\title{
LAPLACE TRANSFORM OF CERTAIN FUNCTIONS WITH APPLICATIONS
}

\section{ASLAM CHAUDHRY}

(Received 21 June 1993)

\begin{abstract}
The Laplace transform of the functions $t^{v}(1+t)^{\beta}$, $\operatorname{Re} v>-1$, is expressed in terms of Whittaker functions. This expression is exploited to evaluate infinite integrals involving products of Bessel functions, powers, exponentials, and Whittaker functions. Some special cases of the result are discussed. It is also demonstrated that the famous identity $\int_{0}^{\infty} \sin (a x) / x d x=\pi / 2$ is a special case of our main result.
\end{abstract}

Keywords and phrases. Laplace transforms, probability density functions, Whittaker functions.

2000 Mathematics Subject Classification. Primary 60E05.

1. Introduction. The Laplace transform of the functions $t^{\nu}(1+t)^{\beta}$, Rev $>-1$, can be expressed in terms of the confluent hypergeometric function $\psi$ [4, p. 268]. Some special cases of the Laplace transform of these functions are listed in the literature [2, 3]. For $\beta=-1$, see [2, p. 137] and for $\beta=v,-v+(1 / 2)$, see [2, p. 319]. In this paper, we exploit the relationship between the Whittaker and the confluent hypergeometric functions to express the Laplace transform of certain functions in terms of the Whittaker functions. Some useful special cases, which do not seem to be listed in the literature, are discussed.

The representation of the Laplace transform of these functions in terms of Whittaker functions is further exploited to evaluate infinite integrals involving products of Bessel functions, powers, exponentials, and Whittaker functions. Some special cases are discussed. For example, it is proved that

$$
\int_{0}^{\infty} x^{\alpha-1} J_{\alpha}(a x) d x=\frac{\Gamma(\alpha)}{2}\left(\frac{2}{a}\right)^{\alpha}, \quad \operatorname{Re} \alpha>0, a>0,
$$

which generalizes the famous identity $\int_{0}^{\infty} \sin (a x) / x d x=\pi / 2$, Re $a>0$.

\section{Main Results}

LEMMA 2.1. Let $\mathrm{L}$ be the Laplace transform operator. Then,

$$
\mathrm{L}\left\{t^{v}(1+t)^{\beta} ; x\right\}=\Gamma(\nu+1) x^{-(\beta+v+2) / 2} e^{x / 2} W_{(\beta-v) / 2,(\beta+v+1) / 2}(x) \quad(v>-1) .
$$

Proof. According to [4, p. 268],

$$
\int_{0}^{\infty} e^{-x t} t^{\alpha-1}(1+t)^{\gamma-\alpha-1} d t=\Gamma(\alpha) \psi(\alpha, \gamma ; x) \quad(\alpha>0) .
$$


Substituting $\alpha=v+1$ and $\gamma=v+\beta+2$ in (2.2), we get

$$
\mathrm{L}\left\{t^{v}(1+t)^{\beta} ; x\right\}=\Gamma(v+1) \psi(v+1, v+\beta+2 ; x) \quad(v>-1) .
$$

However, according to [1, p. 264],

$$
\psi(\nu+1, v+\beta+2 ; x)=x^{-(\beta+v+2) / 2} e^{x / 2} W_{(\beta-\nu) / 2,(\beta+\nu+1) / 2}(x) .
$$

From (2.3) and (2.4), the lemma follows.

COROLlary 2.1. We have

$$
\mathrm{L}\left\{t^{n}(1+t)^{m} ; x\right\}=(n !)(m !) x^{-(n+m+1)} G_{m}^{n}(x),
$$

where $G_{m}^{n}(x)$ are the polynomials defined by

$$
G_{m}^{n}(x)=\sum_{k=0}^{m}\left(\begin{array}{c}
m+n-k \\
n
\end{array}\right) \frac{x^{k}}{k !} .
$$

Proof. This follows from the lemma when we substitute $v=n, \beta=m$ and use the fact that

$$
G_{m}^{n}(x)=\frac{1}{m !} e^{x / 2} x^{a} W_{b, a+1 / 2}(x), \quad a=\frac{m+n}{2}, b=\frac{m-n}{2} .
$$

In particular, when we take $n=0$ in (2.5), we get

$$
\mathrm{L}\left\{(1+t)^{m} ; x\right\}=m ! x^{-m-1} e_{m}(x)
$$

where

$$
e_{m}(x)=\sum_{k=0}^{m} \frac{x^{k}}{k !}
$$

REMARK. It should be noted that the representation of the Laplace transform in terms of the $G$-polynomials is interesting and does not seem to be available in the present form in the literature. This representation could be useful for the research workers in probability theory when $f(t)=C t^{v}(1+t)^{\beta}$ is considered as a probability density function.

COROLLARY 2.2. We have

$$
\mathrm{L}\left\{t^{v}(1+t)^{v} ; x\right\}=\frac{\Gamma(v+1)}{\sqrt{\pi}} x^{-(1 / 2) v} e^{x / 2} K_{(v+1) / 2}\left(\frac{x}{2}\right) \quad(\operatorname{Re} v>-1) .
$$

Proof. This follows from Lemma 2.1 when we take $\beta=v$ and use the relation [4, p. 279].

COROLlary 2.3. See [2, p. 137]

$$
\mathrm{L}\left\{(1+t)^{\beta} ; x\right\}=x^{-\beta-1} e^{x} \Gamma(\beta+1, x) .
$$

Proof. This follows from Lemma 2.1 when we take $v=0$ and use the fact that

$$
W_{\beta / 2,(\beta+1) / 2}(x)=x^{-\beta / 2} e^{x / 2} \Gamma(\beta+1, x) .
$$


THEOREM 2.1. Let $\operatorname{Re} v>-1$, $\operatorname{Re} \mu>0$, and $p>0$. Then,

$$
\begin{aligned}
\int_{0}^{\infty} x^{\nu-1} e^{x / 2} & W_{\alpha, \alpha+v+1 / 2}(x) J_{2 \alpha+2 \mu+2 v}(2 \sqrt{p x}) d x \\
& =\frac{\Gamma(2 \mu)}{\Gamma(\nu+1)} p^{(v-1) / 2} e^{p / 2} W_{(2 \alpha-2 \mu+v+1) / 2,(2 \alpha+2 \mu+v) / 2}(p) .
\end{aligned}
$$

Proof. Let us define a function $f(t)$ by

$$
f(t)=t^{v}(1+t)^{2 \alpha+v} \quad(\operatorname{Re} v>-1) .
$$

Replacing $t$ by $1 / t$ in (2.14) and multiplying both sides by $t^{2 \mu+2 v+2 \alpha-1}$, we get

$$
t^{2 \mu+2 v+2 \alpha-1} f\left(\frac{1}{t}\right)=t^{2 \mu-1}(1+t)^{2 \alpha+v} \quad(\operatorname{Re} \mu>0) .
$$

By using the property [2, p. 132] of the Laplace transformation, we get from (2.15) that

$$
\int_{0}^{\infty} t^{v-1} e^{t / 2} W_{\alpha, \alpha+v+1 / 2}(t) J_{2 \alpha+2 \mu+2 v}(2 \sqrt{p t}) d t=\frac{p^{v+v+\alpha}}{\Gamma(v+1)} \mathrm{L}\left\{t^{2 \mu-1}(1+t)^{2 \alpha+v} ; p\right\} .
$$

However, according to Lemma 2.1, we have

$$
\mathrm{L}\left\{t^{2 \mu-1}(1+t)^{2 \alpha+v} ; p\right\}=\Gamma(2 \mu) p^{-(2 \mu+2 \alpha+v+1) / 2} e^{p / 2} W_{(2 \alpha-2 \mu+v+1) / 2,(2 \alpha+2 \mu+v) / 2}(p) .
$$

From (2.16) and (2.17), the theorem follows.

COROllary 2.4. We have

$$
\begin{aligned}
\int_{0}^{\infty} t^{\mu-1 / 2} e^{t / 2} K_{v+1 / 2} & \left(\frac{t}{2}\right) J_{2 \mu+2 v}(2 \sqrt{p t}) d t \\
& =\sqrt{\pi} \frac{\Gamma(2 \mu)}{\Gamma(v+1)} p^{(v-1) / 2} e^{p / 2} W_{(1+v-2 \mu) / 2,(v+2 \mu) / 2}(p) .
\end{aligned}
$$

Proof. This follows from the theorem when we take $\alpha=0$ and use the fact [4, p. 279] that

$$
W_{0, v}(t)=\sqrt{\frac{t}{\pi}} K_{v}\left(\frac{t}{2}\right)
$$

COROLlary 2.5. We have

$$
\int_{0}^{\infty} x^{\alpha-1} J_{\alpha}(a x) d x=\frac{\Gamma(\alpha)}{2}\left(\frac{2}{a}\right)^{\alpha} \quad(\operatorname{Re} \alpha>0) .
$$

Proof. This follows from (2.18) when we take $v=0, \mu=\alpha / 2, p=a^{2} / 4$ and use the transformation $t=x^{2}$.

In particular, when $\alpha=1 / 2$ in (2.20), we have

$$
\sqrt{\frac{2}{a \pi}} \int_{0}^{\infty} \frac{\sin (a x)}{x} d x=\sqrt{\frac{\pi}{2 a}} \quad(\operatorname{Re} a>0),
$$

which implies that (see [3, p. 405, (3.721) (1)])

$$
\int_{0}^{\infty} \frac{\sin (a x)}{x} d x=\frac{\pi}{2}, \quad(\operatorname{Re} a>0) .
$$


REMARK. The result (2.20) can be regarded as a generalization of the famous identity (2.22) occurring in a variety of engineering problems. Moreover, using (2.20) and the identity [2, p. 148, (11)], we get a new integral formula

$$
\begin{gathered}
\int_{0}^{\infty} x^{\alpha-2 v+1} J_{\alpha}(a x) \ln x d x=\left(\frac{2}{a}\right)^{\alpha-2 v} \frac{\Gamma(\alpha-v+1)}{a^{2} \Gamma(v)}\left[\psi(\alpha-v+1)+\psi(v)-\ln \left(\frac{a^{2}}{4}\right)\right], \\
(a>0, v>0, \alpha-v+1>0),
\end{gathered}
$$

of product of logarithmic and Bessel functions. Several special cases of (2.23) can be listed. In particular, for $a=2$ and $v=1$ in (2.23), we get

$$
\int_{0}^{\infty} x^{\alpha-1} J_{\alpha}(2 x) \ln x d x=\frac{\Gamma(\alpha)}{4}[\psi(\alpha)-\gamma] \quad(\alpha>0),
$$

where $\gamma$ is the Euler constant [3, p. 946]. The substitution $\alpha=1$ in (2.24) leads to the integral representation

$$
\gamma=-2 \int_{0}^{\infty} J_{1}(2 x) \ln x d x
$$

of the Euler constant. If we substitute $\alpha=v=1$ in (2.23), the classical result [3, p. 767 (6.772)(2)],

$$
\int_{0}^{\infty} J_{1}(a x) \ln x d x=-\frac{1}{a}\left[\gamma+\ln \left(\frac{a}{2}\right)\right],
$$

is recovered.

Acknowledgements. The author is indebted to King Fahd University of Petroleum and Minerals and the ICTP for their support. Valuable comments of the referee are appreciated.

\section{REFERENCES}

[1] A. Erdelyi, W. Magnus, F. Oberhettinger, and F. G. Tricomi, Higher Transcendental Functions. Vols. I, II, McGraw-Hill Book Company, Inc., New York, Toronto, London, 1953. MR 15,419i. Zbl 542.33001.

[2] _ Tables of Integral Transforms. Vol. I, McGraw-Hill Book Company, Inc., New York, Toronto, London, 1954. MR 15,868a. Zbl 055.36401.

[3] I. S. Gradshteyn and I. M. Ryzhik, Table of Integrals, Series, and Products, Academic Press [Harcourt Brace Jovanovich, Publishers], New York, London, Toronto, 1980. MR 81g:33001. Zbl 521.33001.

[4] N. N. Lebedev, Special Functions and their Applications, Dover Publications, Inc., New York, 1972. MR 50 2568. Zbl 271.33001.

[5] G. N. Watson, A Treatise on the Theory of Bessel Functions, Cambridge University Press, Cambridge, 1966. MR 6,64a. Zbl 174.36202.

Chaudhry: Department of Mathematical Sciences, King Fahd University of PetroleUM AND MINERALS, DHAHRAN, SAUDI ARABIA

E-mail address: mas 1 am@kfupm. edu. sa 


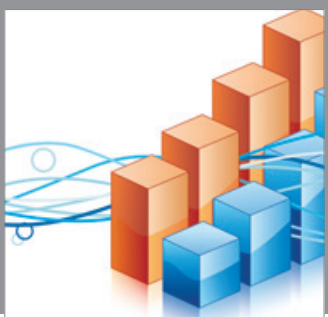

Advances in

Operations Research

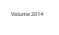

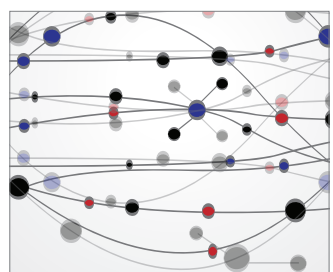

\section{The Scientific} World Journal
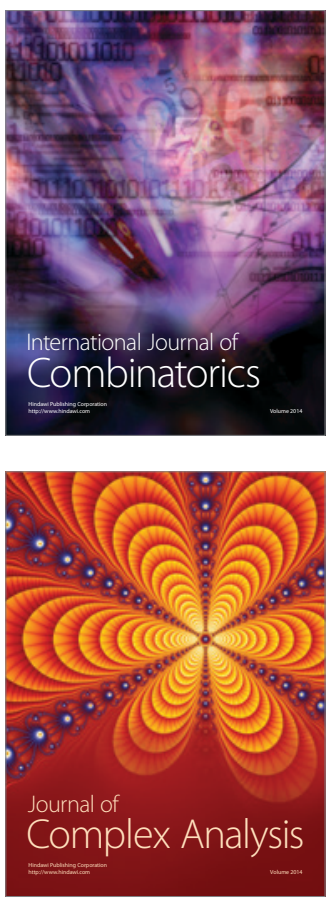

International Journal of

Mathematics and

Mathematical

Sciences
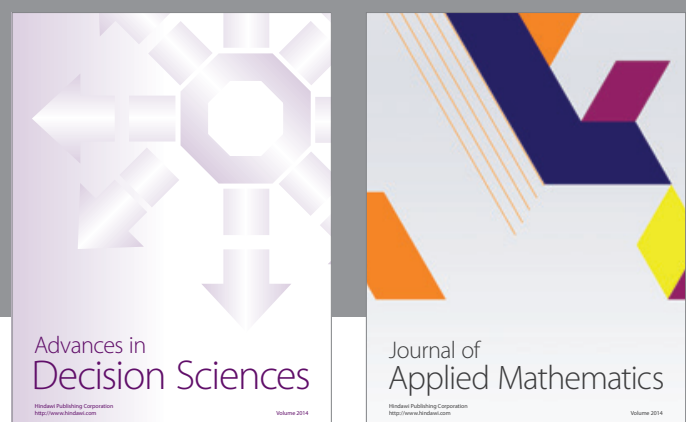

Journal of

Applied Mathematics
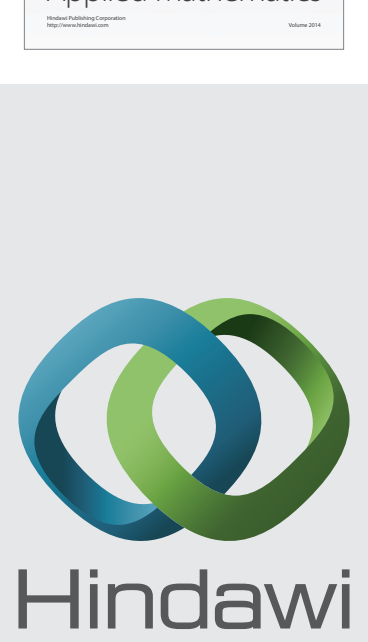

Submit your manuscripts at http://www.hindawi.com
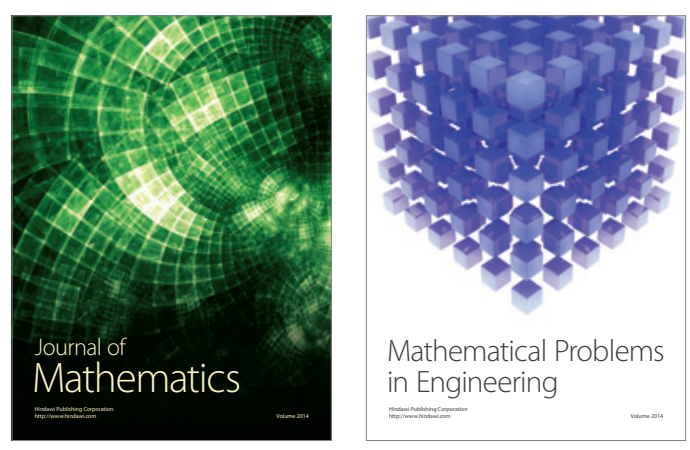

Mathematical Problems in Engineering
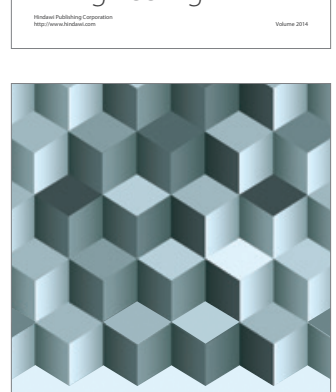

Journal of

Function Spaces
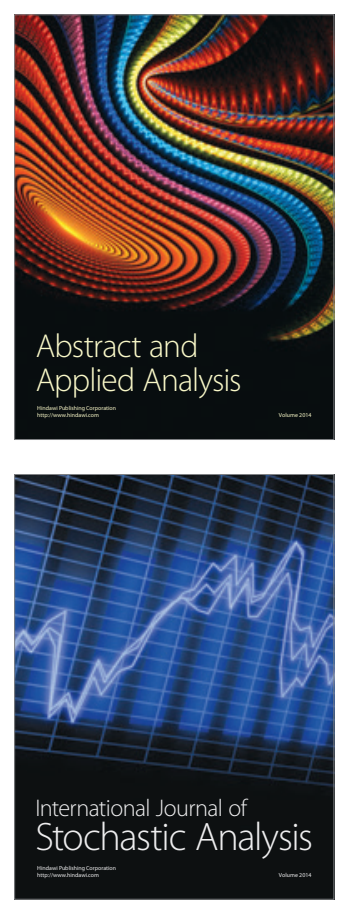

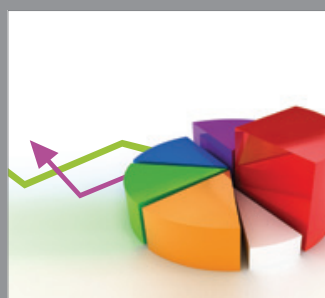

ournal of

Probability and Statistics

Promensencen
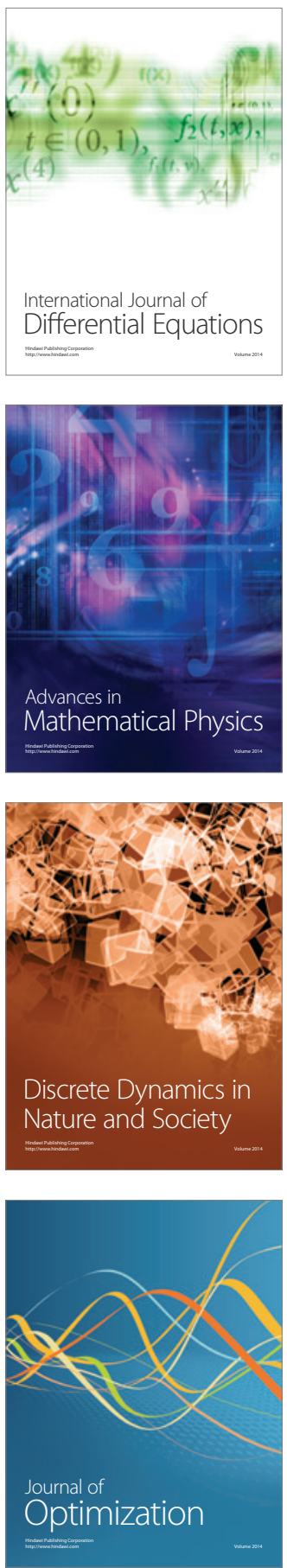